\title{
ASSESSMENT OF CONTINENTAL HYDROSPHERE LOADING USING GNSS MEASUREMENTS
}

\author{
Michał Zygmunt, Marcin Rajner, Tomasz Liwosz \\ Warsaw University of Technology, \\ Department of Geodesy and Geodetic Astronomy, \\ PI. Politechniki 1, PL 00-661 Warsaw, Poland
}

\begin{abstract}
Presented paper is dedicated to problems of deformation of the Earth's crust as a response to the surface loading caused by continental waters. The aim of this study was to specify areas particularly vulnerable to studied deformation and to compare calculated and observed displacements. Information of the continental water volume was taken from the WaterGAP Global Hydrological Model. Calculated values of the deformations were verified with the results obtained with programs SPOTL and grat. Vertical deformations were almost 10 times higher than the deformation in the horizontal plane, for which reason later part of the paper focuses on the former. In order to check agreement of the calculated and observed deformation 23 stations of International GNSS Service (IGS) were selected and divided into three groups (inland, near the shoreline and islands). Before comparison outliers and discontinuities were removed from GNSS observations. Modelled and observed signals were centred. The analysed time series of the vertical displacements showed that only for the inland stations it is possible to effectively remove displacements caused by mass transfer in the hydrosphere. For stations located in the coastal regions or islands, it is necessary to consider additional movement effects resulting from indirect ocean tidal loading or atmosphere loading.
\end{abstract}

Keywords: Load Love Numbers, Green's functions, WGHM, GNSS, Crustal deformation

\section{Introduction}

Recorded time series of the reference station positions, continuous registering satellite observations are influenced with interferences from a number of geodynamic phenomena, such as: precession and nutation, the movements of tectonic plates, deformations caused by tidal forces or polar motion. Understanding the mechanisms of these phenomena with the development of survey instruments made it possible to obtain the accuracy of the position determination on the surface of the Earth with an error of less than $1 \mathrm{~mm}$. Section 7.2 of the International Earth Rotation and Reference System Service Conventions (Petit \& Luzum, 
2010), devoted to the movements of the Earth's crust as the consequences of loading it's surface by the masses of the atmosphere, oceans and terrestrial hydrosphere, contains a recommendation not to consider these effects in calculations, because of the very poor knowledge of the models of these phenomena, although they were subject of studies in the past (van Dam et al., 2001; Tervo et al., 2006).

The contents of this paper is devoted to calculating crustal deformation caused by the hydrosphere loading. Particularly to determine areas prone to this type of deformation and determine the convergence between calculated and observed deformation at selected GNSS stations. It is necessary to know the quantity of hydrosphere masses that are loading the surface of the Earth and the analytical description of the response to the load. Modelling of deformation is possible with geodynamic parameters such as Load Love Numbers and Green's functions derived for a specific Earth model. The hydrosphere masses can be determined using the data in hydrological models. Such model should include water resources contained within calm and flowing waters, groundwater and water stored in the form of snow. This is needed due to the quantitative changes related to the dynamics of the hydrological cycle.

Taking into account the results of this research along with the post-processing of satellite observations will help to increase the accuracy of position determination (mainly the vertical component), and in the future will help to improve the definition of the reference systems.

\section{Earth's crust deformations}

Dimensionless numerical values that defines the response of the Earth elastic model to the effect of load, were originally defined by Love, Shida and Matsuyama and they are called from the name of the first researcher Load Love Numbers (LLN; Benavent et al., 2005). Numerical values describing the deformations, depend on the internal structure of the Earth and particularly on the structure of the crust and mantle in regions close to the loading mass (Benavent et al., 2005). For this reason, the LLN must be calculated separately for each Earth model (Farrell, 1972).

Green's functions can be used to describe the displacement of points located both on the Earth's surface and in its interior. In the latter, used LLN should be first calculated for points inside the body. They are formed by summing weighted LLN and depend on the distance from the centre of the body (a) and angular distance $(\theta)$ from loading mass. To calculate crustal deformation caused by hydrosphere loading for GNSS stations, average radius of the Earth ( $a$ - radius of the spherical Earth) should be used (Farrell, 1972).

Expanding Legendre polynomial to order of 10000 allows to calculate Green's functions with precision corresponding to the real crustal deformation. Adoption of too low polynomial order cause problems with displacements calculation in coastal areas (Gibbs effect). With large enough polynomial order LLN (i.e. $h_{n}^{\prime}, l_{n}^{\prime}$ and $k_{n}^{\prime}$ ) become constants (Farrell, 1972).

Green's functions calculated for different Spherical Non-Rotating Elastic Isotropic (SNREI) model show discrepancies only for a small angular distance $\theta$ between the loading mass and the observation point. This is caused due to significant differences in the crust and upper mantle. Van Dam et al. (2003) made a comparison between Green's functions derived for four SNREI models of the Earth, and differences of radial deformations (the vertical direction) were always less than $0.04 \mathrm{~mm}$, which indicates that the choice of LLN for SNREI model of the Earth has no significant impact on calculated effect of the load (Benavent et al., 2005).

Exact values of the Love Load Numbers, depending on $n$ order of expanded Legendre polynomial and Green's function coefficients used to calculate deformation, depending on 
the spherical distance to observation point, were taken for the Earth model 'A', developed jointly by Gutenberg and Bullen (Farrell, 1972).

\section{WaterGAP Global Hydrology Model (WGHM)}

"Water - Global Assessment and Prognosis" hydrological data model (WaterGAP) was developed by the Centre of Environmental System Research at the University of Kassel, Germany, to estimate volume of water resources (Döll et al., 2001).

Part of it, named WaterGAP Global Hydrology Model (WGHM), contains information of water quantity for continental area, without Antarctica and Greenland (Güntner et al., 2007).

WGHM data is divided to 259200 cells with spatial resolution of $0.5^{\circ} \times 0.5^{\circ}$. Each of them, that contains land area of at least $5^{\prime} \times 5^{\prime}$ (approximately $86 \mathrm{~km}^{2}$ ), is considered as a computational cell, for which specified information about the Earth's land surface and fresh water volume are determined (Döll et al., 2003).

Modelled continental hydrosphere include water in natural lakes, artificial reservoirs, wetlands, groundwater, land and snow cover (Hunger \& Döll, 2008).

\section{Calculation of the Earth's crust deformation}

\subsection{Theoretical assumptions of the modelled deformation}

Below are given the formulas suggested by the Farrell (1972), for calculating the deformation in the direction of the plumb line $(u)$ and in the direction of meridian and prime vertical $(n, e)$, following formulae are used in this study,

$$
\begin{gathered}
u=\rho \cdot \iint_{\text {Earth }} G_{\text {radial }}\left(\left|r-r^{\prime}\right|\right) \cdot H\left(r^{\prime}\right) \cdot d A \\
\left\{\begin{array}{l}
n \\
e
\end{array}\right\}=\rho \cdot \iint_{\text {Earth }} G_{\text {tangent }}\left(\left|r-r^{\prime}\right|\right) \cdot H\left(r^{\prime}\right) \cdot d \alpha \cdot\left\{\begin{array}{l}
-\cos (\alpha) \\
-\sin (\alpha)
\end{array}\right\}
\end{gathered}
$$

where $\rho$ is water density, $G_{\text {radial }}, G_{\text {tangent }}$ are Green's function coefficients, describing response of the Earth's crust to the loading in the direction of plumb line and the horizontal plane respectively, $\left|r-r^{\prime}\right|$ means spherical distance between point of observation and a loading mass (spherical figure) $H\left(r^{\prime}\right)$ express average water column height of a loading mass (spherical figure), $d \alpha$ is area of the spherical figure and $\alpha$ stands for azimuth of a loading mass (spherical figure).

The analysis performed by Rajner and Liwosz (2011) for GNSS station in Józefosław, Poland, showed a clear dependence between range of loading mass taken into account and resulted displacement values. Calculations can't rely solely on local or regional loading masses located in the vicinity of the station. Complete displacement values can be obtained only by taking into account the loading mass for whole Earth. For this reason, the calculation performed in the paper were carried out up to the antipodes of each of the calculated points.

Key features of the WGHM model related to the results of the calculations are

- quantitative data expressed as an equivalent height of fresh water column ( $\rho=$ $1000 \mathrm{~kg} / \mathrm{m}^{3}$ ),

- contains information about the entire continental water (including groundwater), but excluding Antarctica and Greenland,

- time range of data: $01.2002-12.2012$ (132 months),

- time resolution: 1 month,

- spatial resolution: $0.5^{\circ} \times 0.5^{\circ}$. 


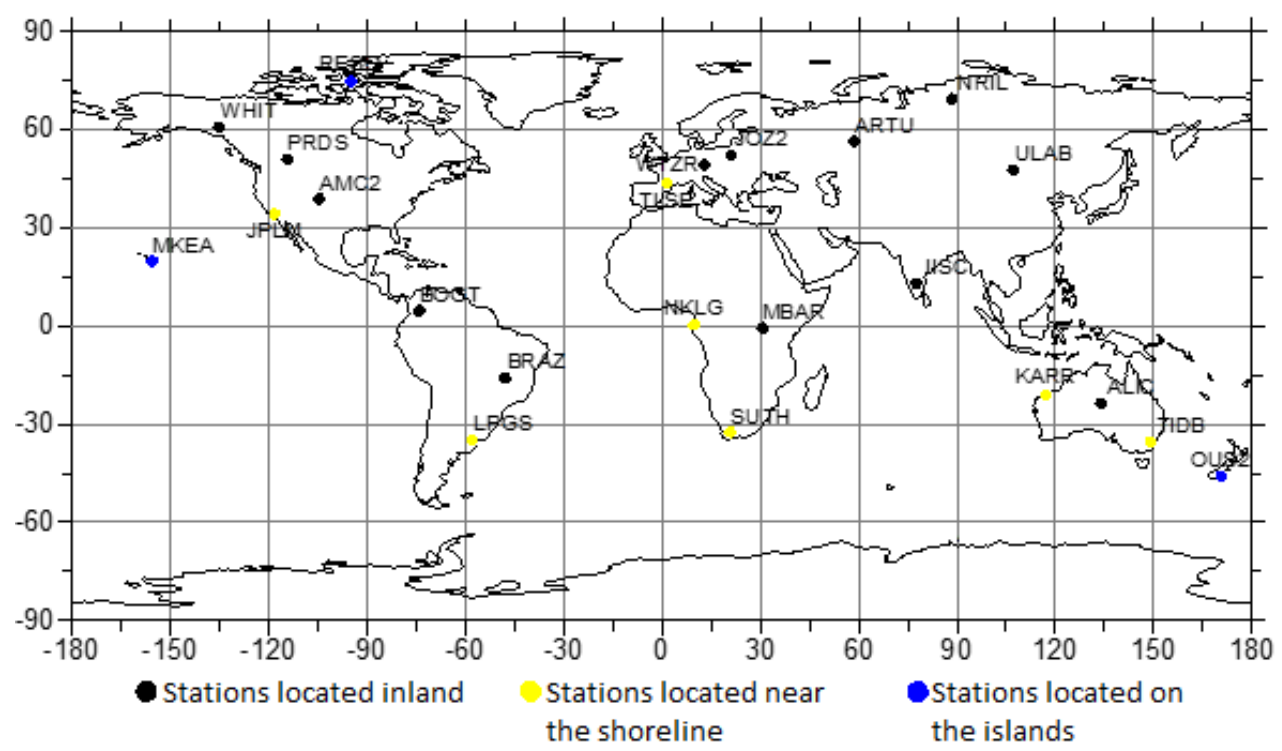

Fig. 1. The location of the selected control stations

Individual segment influence on displacement is calculated by numerical integration of spherical triangles and trapezoids. The performed calculations used approach with constant number of sectors in each zone.

\subsection{Integration figures size determination}

Preliminary analysis, concerning integration parameters, were conducted for station JOZ2 located in central Europe, and a point located in the delta of the Amazon river. For an expected values of the deformations at the specified coordinates were taken the results obtained with SPOTL (Some Programs for Ocean-Tide Loading, Agnew, 2012). In the analysis, three sizes of width of figures of integration were taken into consideration $\left(5^{\circ}, 3^{\circ}, 1^{\circ}\right)$ along with three basic steps between adjacent zones $(2000 \mathrm{~m}, 1000 \mathrm{~m}, 500 \mathrm{~m})$, for a total of nine pairs of parameters.

Obtained results led to following assumptions when calculating Earth's crust deformations

- as a reference surface spherical Earth was adopted,

- width of the elementary figures for numerical integration: $3^{\circ}$,

- basic distance of integration: $500 \mathrm{~m}$.

Parameters related to the size of spherical figures were adopted as a compromise between time of executing calculations and obtained results of deformation discrepancies.

\section{Results of modelling the deformation of the Earth's crust}

\subsection{Selection of control points}

Control points for calculated deformation should be distributed evenly over the entire Earth and variations of their coordinates should be known with high accuracy. Size of the crustal displacement was checked using satellite observations made at 23 selected stations that are part of the IGS network, dividing them into three classes

- 13 stations located inland (distance from the shoreline $>200 \mathrm{~km}$ ),

- 7 stations located near shoreline (distance from the shoreline $<200 \mathrm{~km}$ ),

- 3 stations located on an islands.

The location of the selected stations and their belonging to a specific group is given on Fig. 1 . 
Tab. 1. Basic features of the programs used to compare calculated deformations

\begin{tabular}{|l|c|c|c|}
\hline Feature & MZ & SPOTL & grat \\
\hline Number of sectors in zone: & 120 & $150-360$ & $100-360$ \\
Width of sector: & $3^{\circ}$ & $2.4^{\circ}-1^{\circ}$ & $3.6^{\circ}-1^{\circ}$ \\
Integral interval (distance): & $500 \mathrm{~m}$ & vary & vary \\
No. of base (source) values of Green's function & 50 & 438 & 85 \\
\hline
\end{tabular}

\subsection{Analysis of the accuracy of the numerical integration}

Size of the integration figures, the way of division zones to segments and the spherical distances for which the Green's function are interpolated can affect the results of calculated deformations. This paragraph was devoted to a comparison between the displacements calculated by different methods. Into account were taken: a program written for the purpose of this study (MZ), a program SPOTL (Agnew, 2012) and program grat (Rajner, 2013). Each of them represent a slightly different approach to the calculation of crustal deformations.

The preliminary analysis on the width of the figures of integration, contained in section 4.2, showed that in the case of the author method, changing the width of the sector doesn't have much significance for the results of calculated deformations. Other programs used an approach, where the number of sectors of each zone depends on the distance from the point for which the deformations are calculated, but is not less than accepted minimum - maximum number of sectors falls in the zone midway to the antipodal point. Other differences in the calculations are related to the distance (interval) of integration and Green's function values for specified spherical distance. In this paper, the author uses the Green's function derived by Farrell (1972), which were determined for 50 spherical distances up to antipodal point. Their values between individual steps are interpolated with cubic spline. In SPOTL values of Green's function include 438 intermediate steps, between which the spacing increases with distance from point of calculation (from $0.01^{\circ}$ to $1^{\circ}$ ). In case of grat the size of the spherical figures are adjusted so that the individual steps of integration correspond to the spherical distance for which Green's function values are calculated (85 steps). Table 1 summarizes the basic parameter of the programs mentioned above, that are affecting the obtained results of calculations. Table 2 summarizes the differences in the calculated displacements for every control station and summarizes the extreme differences of results.

In comparison to the results obtained by the author, the biggest difference was achieved for the station: MBAR, MKEA, NKLG, RESO and WHIT. In all cases where obtained differences exceeded $1 \mathrm{~mm}$ were related to the deformation of the vertical component. The largest discrepancies were related to the stations: MKEA and RESO, where the calculated displacements were up to $1.8 \mathrm{~mm}$ (grat) and $-1.6 \mathrm{~mm}$ (SPOTL), respectively. Displacement values in the direction of the meridian were higher when compared to the results of programs SPOTL and grat by up to $0.5 \mathrm{~mm}$. In the case of deformations in the direction of prime vertical almost the same results were obtained.

It should also be noted the high agreement of the results obtained with SPOTL and grat. Differences in horizontal components, beyond the individual cases (NKLG), were no greater than $0.1 \mathrm{~mm}$. In case of deformations of the vertical component, differences for four stations (MKEA, NKLG, OUS2, RESO) exceeded $1 \mathrm{~mm}$. Among them, the most interesting case in NKLG where the difference between the results obtained with SPOTL and grat was $1.9 \mathrm{~mm}$-- both programs shows deformations in opposite directions. 
Tab. 2. The differences between values of deformation at control stations obtained with programs: (author's) MZ, SPOTL and grat and the extreme differences between deformations obtained with programs: (author's) MZ, SPOTL and grat at the control stations. Values marked in red indicates differences greater than $1 \mathrm{~mm}$

\begin{tabular}{|c|c|c|c|c|c|c|c|c|c|c|}
\hline Station ID & \multicolumn{2}{|c|}{ MZ-SPOTL $[\mathrm{mm}]$} & \multicolumn{3}{|c|}{ MZ-grat [mm] } & \multicolumn{3}{|c|}{ SPOTL-grat [mm] } \\
\hline & $\mathrm{n}$ & $\mathrm{e}$ & $\mathrm{u}$ & $\mathrm{n}$ & $\mathrm{e}$ & $\mathrm{u}$ & $\mathrm{n}$ & $\mathrm{e}$ & $\mathrm{u}$ \\
\hline ALIC & 0.4 & 0.0 & -0.7 & 0.4 & 0.0 & -0.7 & 0.1 & 0.0 & 0.0 \\
AMC2 & 0.2 & 0.0 & -0.2 & 0.2 & -0.1 & -0.1 & 0.0 & -0.1 & 0.1 \\
ARTU & 0.3 & 0.0 & 0.9 & 0.2 & 0.0 & 0.9 & 0.0 & -0.1 & 0.1 \\
BOGT & 0.3 & -0.3 & -0.2 & 0.3 & -0.1 & 0.2 & 0.0 & 0.1 & 0.4 \\
BRAZ & 0.2 & 0.0 & 0.6 & 0.1 & -0.1 & 0.7 & -0.1 & -0.1 & 0.1 \\
DAV1 & 0.1 & 0.0 & 0.2 & 0.1 & 0.0 & 0.2 & 0.0 & 0.1 & 0.0 \\
IISC & -0.1 & 0.2 & 0.2 & 0.0 & 0.2 & 0.6 & 0.1 & -0.1 & 0.5 \\
JOZ2 & 0.2 & -0.1 & 0.6 & 0.2 & 0.0 & 0.6 & 0.0 & 0.2 & 0.1 \\
JPLM & 0.2 & 0.0 & 0.3 & 0.2 & 0.1 & 0.2 & 0.0 & 0.1 & -0.1 \\
KARR & 0.5 & 0.1 & -0.9 & 0.5 & 0.2 & -0.7 & 0.0 & 0.1 & 0.2 \\
KELY & 0.1 & -0.2 & -0.5 & 0.1 & -0.1 & -0.4 & 0.0 & 0.1 & 0.0 \\
LPGS & 0.3 & 0.1 & -0.9 & 0.1 & 0.1 & 0.1 & -0.1 & 0.0 & 1.0 \\
MBAR & 0.1 & 0.1 & 1.1 & 0.1 & 0.1 & 1.3 & 0.0 & 0.0 & 0.1 \\
MKEA & 0.1 & 0.1 & 0.6 & 0.1 & -0.1 & 1.8 & 0.0 & -0.1 & 1.2 \\
NKLG & 0.3 & -0.3 & -0.7 & 0.2 & 0.1 & 1.2 & -0.1 & 0.4 & 1.9 \\
NRIL & 0.3 & 0.0 & -0.2 & 0.4 & 0.1 & 0.4 & 0.0 & 0.1 & 0.6 \\
OUS2 & 0.2 & -0.1 & -0.9 & 0.2 & 0.0 & 0.2 & 0.0 & 0.1 & 1.1 \\
PRDS & 0.2 & -0.1 & 0.7 & 0.2 & 0.0 & 1.0 & 0.0 & 0.1 & 0.3 \\
RESO & 0.1 & -0.2 & -1.6 & 0.2 & -0.1 & -0.2 & 0.0 & 0.1 & 1.5 \\
SUTH & 0.1 & 0.1 & 0.1 & 0.1 & 0.1 & 0.2 & 0.0 & 0.0 & 0.1 \\
TIDB & 0.2 & 0.0 & -0.1 & 0.3 & 0.0 & 0.2 & 0.0 & 0.0 & 0.3 \\
TLSE & 0.3 & 0.2 & 0.0 & 0.4 & 0.2 & 0.4 & 0.0 & -0.1 & 0.4 \\
ULAB & 0.0 & 0.1 & 0.5 & 0.1 & 0.0 & 0.6 & 0.0 & 0.0 & 0.1 \\
WHIT & 0.0 & -0.2 & -1.0 & 0.3 & 0.0 & -0.3 & 0.3 & 0.1 & 0.7 \\
WTZR & 0.2 & 0.1 & 0.2 & 0.3 & 0.1 & 0.3 & 0.1 & 0.0 & 0.1 \\
\hline maX. & 0.5 & 0.2 & 1.1 & 0.5 & 0.2 & 1.8 & & & \\
min. & -0.1 & -0.3 & -1.6 & 0.0 & -0.1 & -0.7 & & & \\
abs. avg. & 0.2 & 0.1 & 0.6 & 0.2 & 0.1 & 0.5 & & & \\
\hline & & & & & & & & \\
\hline
\end{tabular}

Fig. 2 shows the vertical deformation of the station NKLG (a) calculated with programs: (author's) MZ, SPOTL and grat, as well as differences between the results of individual programs (b). Obtained discrepancies are related to the location of the station (located on the coast) and suggest the need to consider additional effects such as atmospheric loading.

\subsection{Continental crust deformation}

Using the adopted size of figures of integration calculations of the deformations were carried out for points distributed over the Earth in a regular grid, at a distance of $5^{\circ}$. Because Earth is under constant load of land's hydrosphere, the study of the crustal deformation as the consequences of this phenomenon should be considered in differential approach. For this purpose, deformations were calculated for two months, specially selected to demonstrate the movement of the Earth's crust as the consequences of long-term changes in land's hydrosphere. Those months were June 2002 and June 2012 and the displacements' 
a)
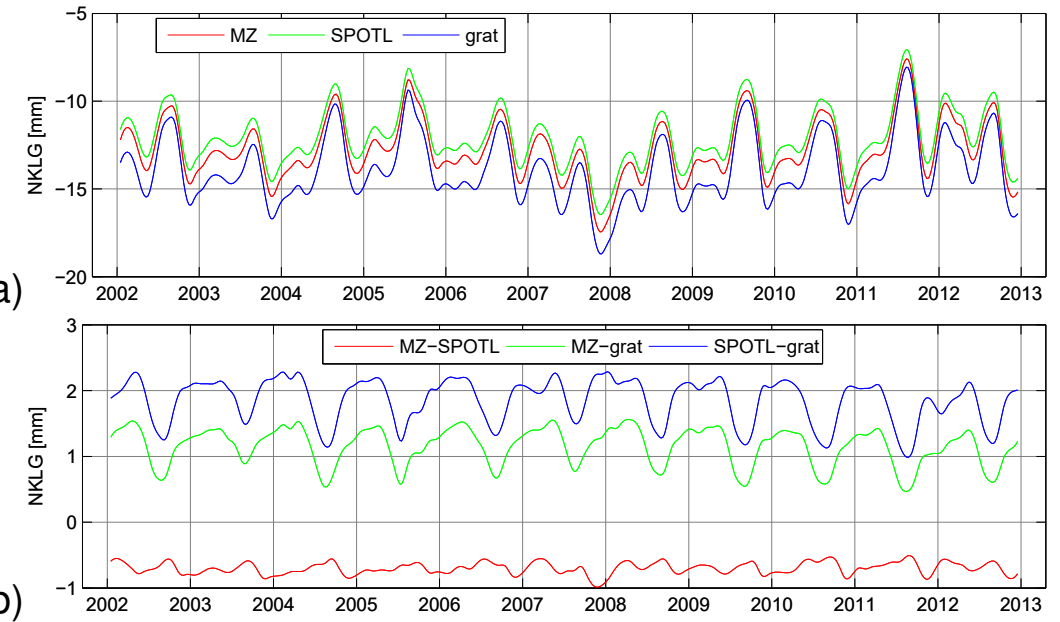

Fig. 2. Calculated vertical displacements of station NKLG in successive epochs (a) and the differences between the results obtained with the programs: (author's) MZ, SPOTL and grat (b)

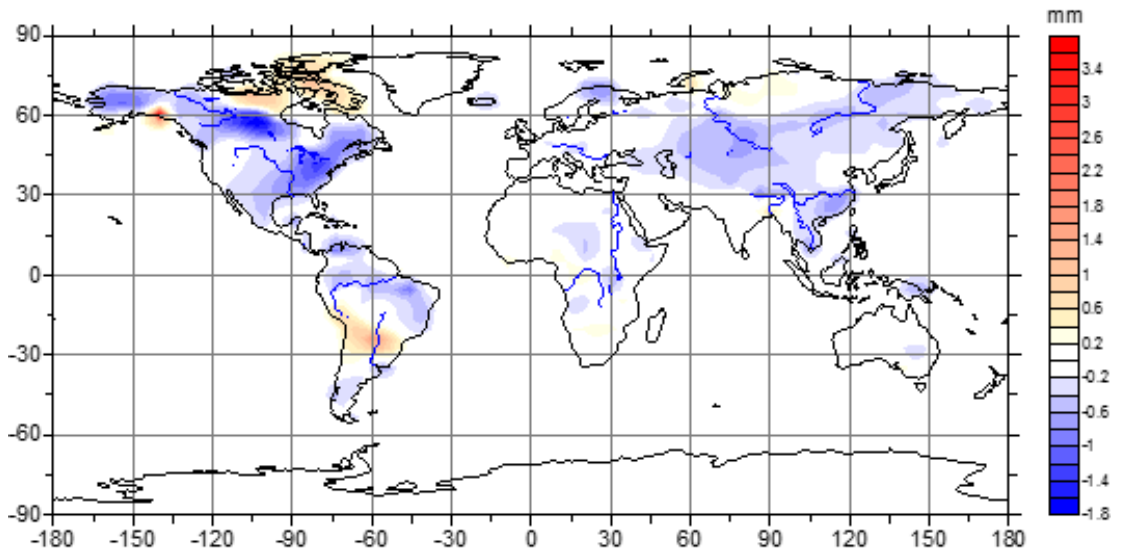

Fig. 3. Crustal displacements in the direction of meridian, which occurred between June 2002 and June 2012

differences are shown in the local coordinate system of each point. Figures 3,4 and 5 contains only the differences between displacements calculated for considered months in the direction of meridian, prime vertical and plumb line, respectively.

Horizontal displacements reaches several fold lower values than the deformation in the vertical direction. Among them, greater values are reached in the direction of meridian.

Long-term deformations presented in Fig. 5 shows a significant lowering of land's surface especially in equatorial climate zone, for which extreme value occurs in the Amazon river area, and reaches a value of $-9 \mathrm{~mm}$ in relation to June 2002. Rising of the Earth's crust occurs over a vast region of North America (average lifting velocity of $2 \mathrm{~mm} / \mathrm{year}$ ) and in the central part of Eurasia.

However, note that the presented deformations have been calculated for a particular month and do not have the characteristic of a trend - e.g. they may be susceptible to local anomalies caused by the local accumulation of continental waters.

\subsection{Preparation of the observed GNSS time series for comparison with the calcu- lated displacements}

Determination of GNSS station displacements require use of models recommended by the IERS Convention as well as IGS products, so the most probable coordinates of the selected 


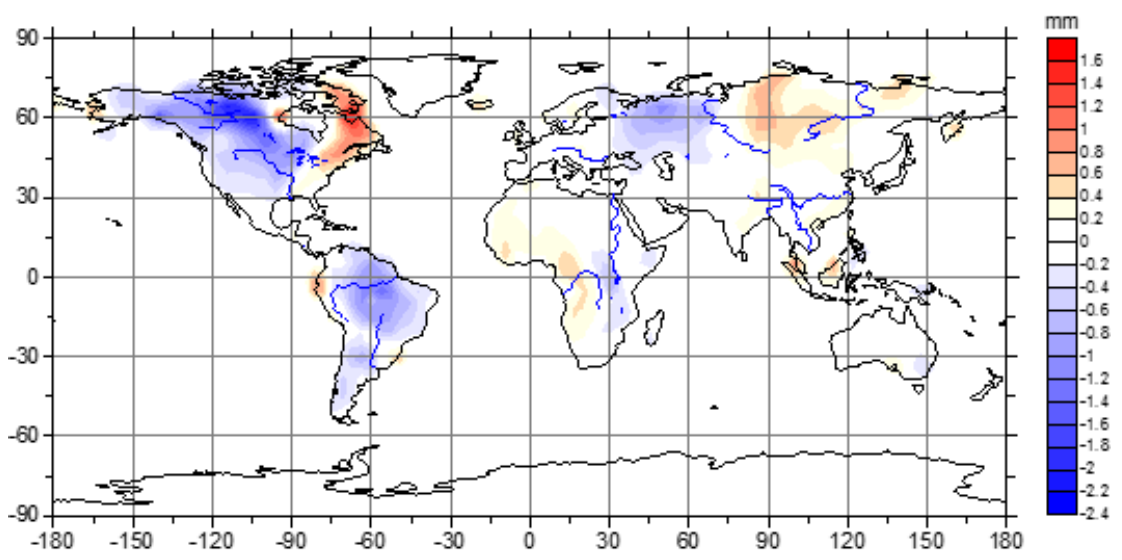

Fig. 4. Crustal displacements in the direction of prime vertical, which occurred between June 2002 and June 2012

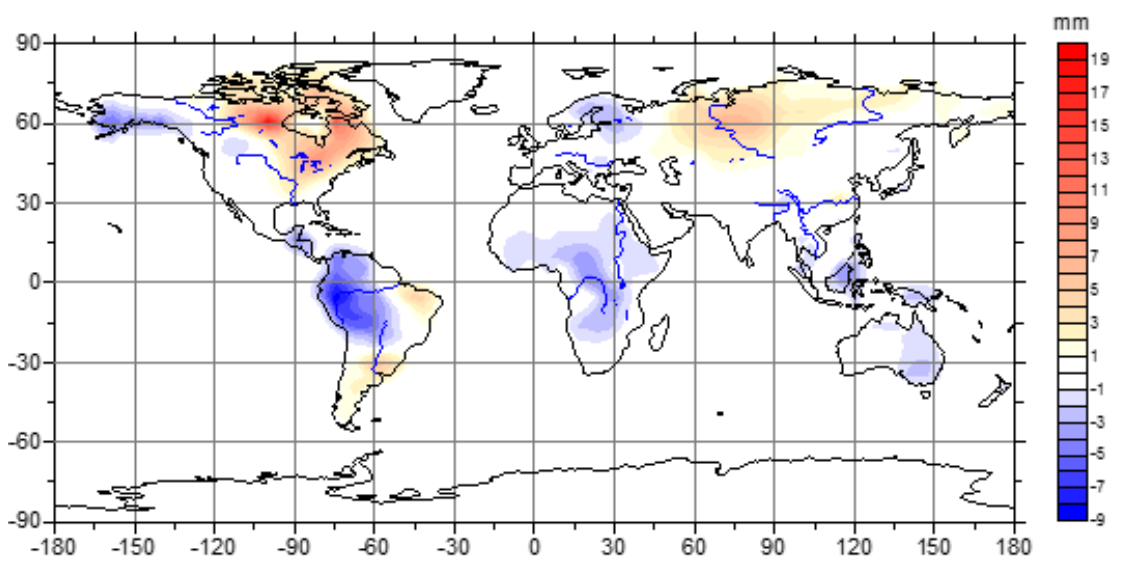

Fig. 5. Crustal displacements in the direction of plumb line, which occurred between June 2002 and June 2012

points of the Earth's crust can be achieved. Calculated coordinates are theoretically affected only by the influence of the effects not included in the IERS Conventions, and thus inter alia include the effect of loading earth's crust by terrestrial hydrosphere. Ocean and atmospheric loading aren't included in GNSS coordinates. It suggest also that the calculated deformations should be smaller than the observed displacements.

Both signals have different time range as WGHM displacements were calculated for whole data in hydrosphere model. GNSS stations displacements were calculated on the basis of weekly solutions of individual stations, however coordinate solutions weren't available for all epochs. Comparison of observed and modelled displacement require use of uniform reference frame, and as one, the local frame of calculated deformations was adopted.

Before using the observed GNSS time series outliers had to be removed, points of discontinuity identified and the trend for each fragment between discontinuities removed. Sets of time series prepared in the following manner were used in the later work.

Figures on the following pages shows centred time series of observed and modelled displacements. Each of them also contains information about signals correlation and their shift, expressed in the number of weeks.

Correlation coefficient was calculated as best fit of both signals. Positive value was obtained if both methods showed displacements in the same direction. Negative, if direction was opposite. Signals lag is calculated for epoch of best correlation coefficient. Positive 
Tab. 3. Features of compared signals for station located inland. The correlation coefficients refers to the observed and modelled vertical displacements; Shift of the signals is expressed in number of weeks; Difference of signals amplitudes were calculated assuming GNSS displacement as reference

\begin{tabular}{|l|r|r|c|}
\hline Station ID & $\begin{array}{l}\text { Correlation } \\
\text { coefficient }\end{array}$ & $\begin{array}{l}\text { Signals shift } \\
\text { [weeks] }\end{array}$ & $\begin{array}{l}\text { Difference of signals } \\
\text { amplitudes [mm] }\end{array}$ \\
\hline ALIC & 0.63 & -12 & 5.2 \\
AMC2 & 0.30 & -22 & 3.3 \\
ARTU & 0.52 & 4 & 6.3 \\
BOGT & 0.44 & -2 & 9.0 \\
BRAZ & 0.87 & -4 & 6.9 \\
IISC & 0.70 & -5 & 5.8 \\
JOZ2 & 0.64 & 1 & 3.4 \\
MBAR & -0.45 & 50 & 3.4 \\
NRIL & 0.61 & 1 & 3.9 \\
PRDS & 0.40 & -8 & 4.1 \\
ULAB & 0.22 & -1 & 7.1 \\
WHIT & 0.56 & -5 & 3.4 \\
WTZR & 0.58 & 1 & 1.5 \\
\hline
\end{tabular}

values were obtained if displacement were firstly observed with GNSS technique. Negative, when displacement were firstly observed in WGHM signal.

Since both signals are not symmetrical, for each of them series of local amplitudes were determined, including positive and negative peaks of the signal. Mean amplitude of given time series was calculated as the average of the peaks absolute values and expressed in millimetres. Differences of signal amplitudes presented in the tables were calculated assuming GNSS displacement as reference.

Coordinates of the stations that belong to the IGS are made available to the public as one of the product of this service (Dow et al., 2009).

\subsection{Deformation on GNSS station located inland}

In general, modelled deformations are in agreement with the observed displacements. Correlation of the 8 of 13 stations were greater than 0.50 . Among them, the best compatibility in terms of values of deformations were obtained for the stations ARTU, JOZ2, NRIL, PRDS, WHIT and WTZR, when the modelled coefficients allow to eliminate most of the observed station's displacements. In case of stations ALIC, AMC2, BOGT, BRAZ and IISC very good correlations of signals were obtained, but modelled values of the displacements are about two times smaller than those observed at these stations. Station MBAR lacks many epochs of observation and should be discarded from further analysis. A special case is the ULAB station located in Mongolia. The time series derived from GNSS observations show a clear vertical movement of the crust that oscillates between values of about $-8 \mathrm{~mm}$ to $8 \mathrm{~mm}$, while displacements derived from hydrological data do not exceed $1 \mathrm{~mm}$. Observed station movement may be caused by the direct effect of atmospheric loading over a wide area. Table 3 summarizes the main characteristics of the analysed signals. To describe the compatibility of the observed and modelled displacement selected correlation coefficient and the mutual shift (lag) of both signals, expressed in number of weeks. Figures 6, 7 and 8 contains displacements graphs of the stations considered in the paragraph. 

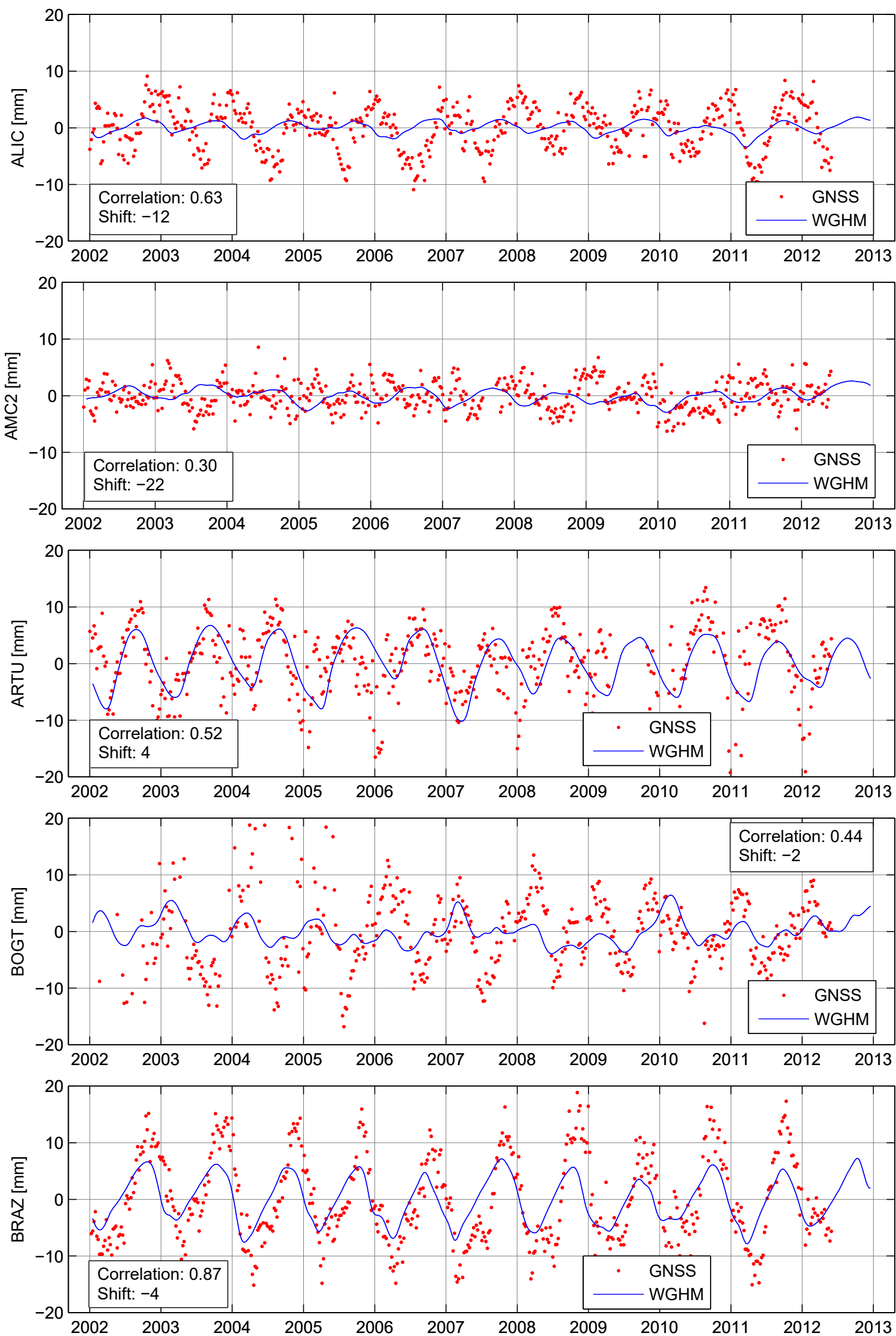

Fig. 6. Vertical displacements of the stations located inland: ALIC, AMC2, ARTU, BOGT, BRAZ. Graphs contains information about correlation of the observed and modelled signals and their mutual shift (lag) expressed in number of weeks 

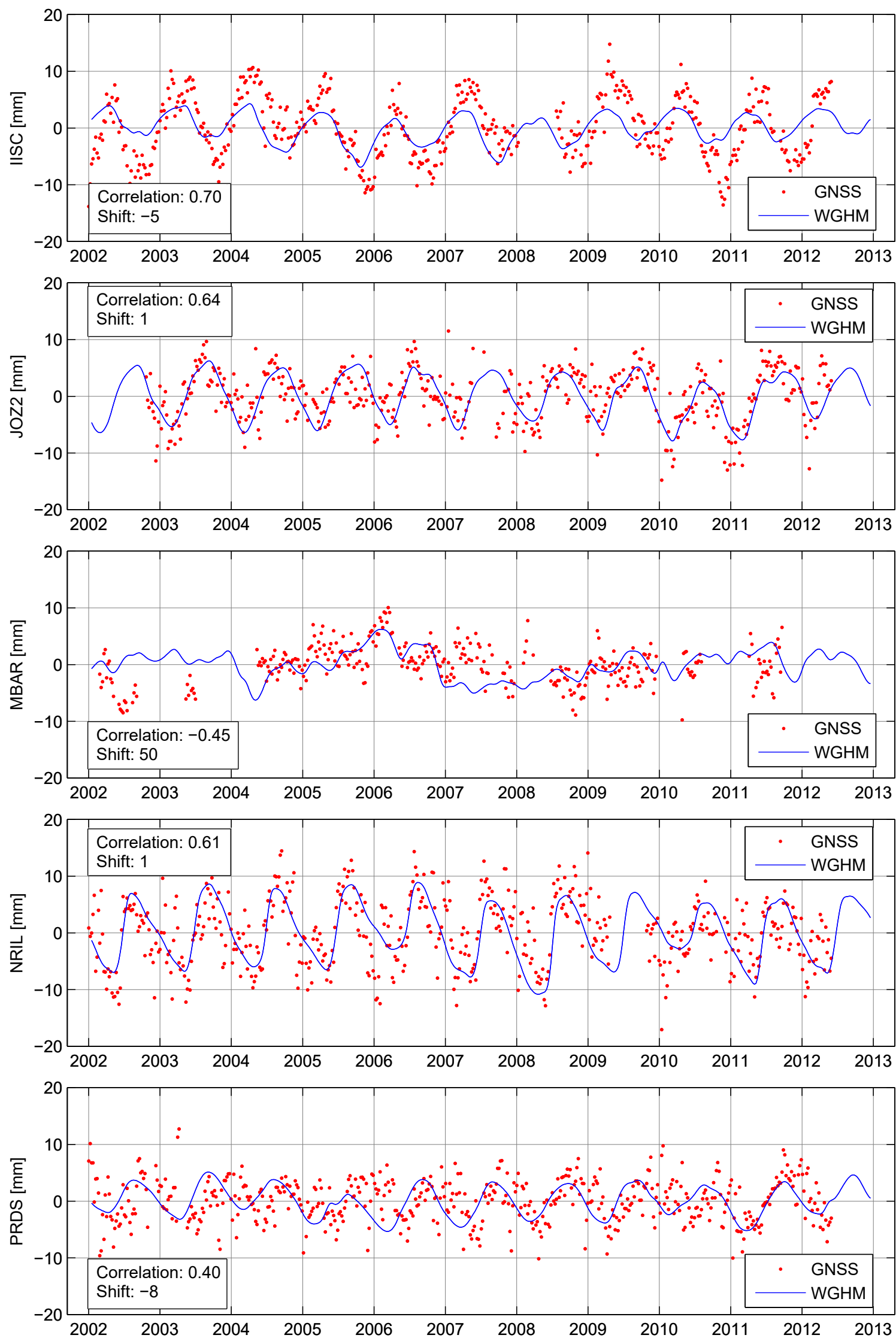

Fig. 7. Vertical displacements of the stations located inland: IISC, JOZ2, MBAR, NRIL, PRDS. Graphs contains information about correlation of the observed and modelled signals and their mutual shift (lag) expressed in number of weeks 

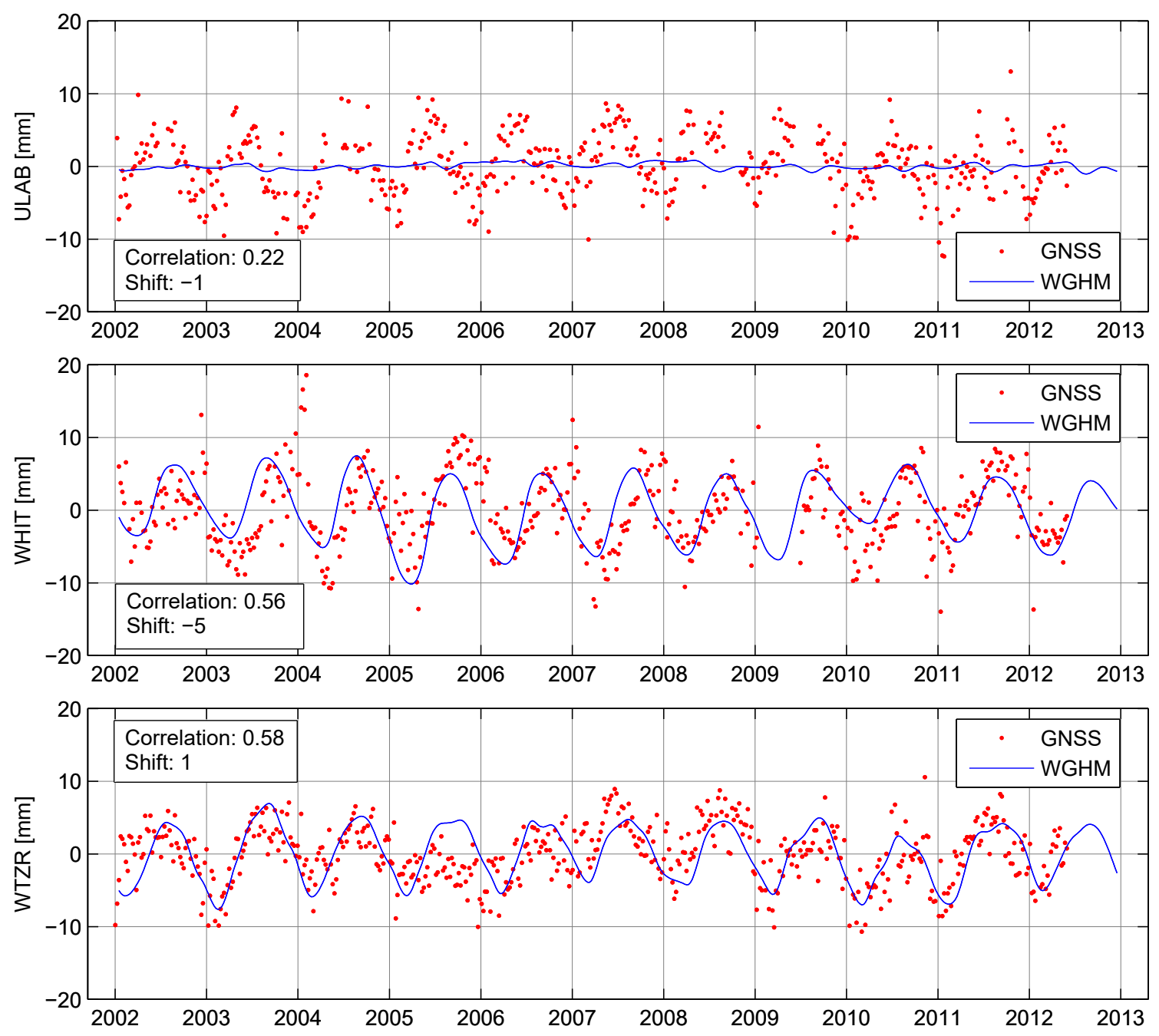

Fig. 8. Vertical displacements of the stations located inland: ULAB, WHIT, WTZR. Graphs contains information about correlation of the observed and modelled signals and their mutual shift (lag) expressed in number of weeks

\subsection{Deformation on GNSS stations located near the shoreline}

Stations located near the shoreline, beyond the displacements resulting from the quantitative changes in land hydrosphere, may be subject to periodic displacements arising as a consequence of indirect ocean tides. Analyses included seven stations located no further than $200 \mathrm{~km}$ from the nearest. The best results of modelled deformations were achieved for TLSE station located in France, in the vicinity of the Bay of Biscay (Atlantic Ocean) and the Mediterranean Sea, with the displacements characteristic for stations located inland. Modelled value of movements can remove almost all observed motion of this station. The influence of the adjacent water masses do not affect station movement, which may be caused by geological substrate. For the rest of the stations in this paragraph calculated displacements were much smaller than observed values. In case of stations: JPLM, NKLG and SUTH achieved very poor results of the signals correlation, which results in a very large shift of both signals. Additionally, in the case of SUTH station, located in South Africa, as in the case of the ULAB station (located inland), calculated vertical displacements does not exceed $1 \mathrm{~mm}$ when the observed movement oscillates between the values of -10 to $10 \mathrm{~mm}$. For remaining stations: KARR, LPGS and TIDB higher correlation coefficient was obtained 
Tab. 4. Features of compared signals for station located inland. The correlation coefficients refers to the observed and modelled vertical displacements; Shift of the signals is expressed in number of weeks; Difference of signals amplitudes were calculated assuming GNSS displacement as reference

\begin{tabular}{|l|r|r|c|}
\hline Station ID & $\begin{array}{l}\text { Correlation } \\
\text { coefficient }\end{array}$ & $\begin{array}{l}\text { Signals shift } \\
\text { [weeks] }\end{array}$ & $\begin{array}{l}\text { Difference of signals } \\
\text { amplitudes [mm] }\end{array}$ \\
\hline JPLM & -0.35 & -8 & 5.7 \\
KARR & 0.68 & -12 & 5.5 \\
LPGS & 0.59 & -10 & 7.1 \\
NKLG & -0.40 & 44 & 5.4 \\
SUTH & -0.69 & -42 & 6.5 \\
TIDB & 0.41 & -3 & 5.8 \\
TLSE & 0.52 & 1 & 1.8 \\
\hline
\end{tabular}

Tab. 5. Features of compared signals for station located inland. The correlation coefficients refers to the observed and modelled vertical displacements; Shift of the signals is expressed in number of weeks; Difference of signals amplitudes were calculated assuming GNSS displacement as reference

\begin{tabular}{|l|r|r|c|}
\hline Station ID & $\begin{array}{l}\text { Correlation } \\
\text { coefficient }\end{array}$ & $\begin{array}{l}\text { Signals shift } \\
\text { [weeks] }\end{array}$ & $\begin{array}{l}\text { Difference of signals } \\
\text { amplitudes [mm] }\end{array}$ \\
\hline MKEA & 0.22 & -31 & 4.9 \\
OUS2 & 0.37 & 1 & 5.5 \\
RESO & -0.36 & 15 & 3.7 \\
\hline
\end{tabular}

(less than 0.50 only for TIDB station). Table 4 summarizes the main characteristics of the analysed signals. To describe the compatibility of the observed and modelled displacements selected correlation coefficient and mutual shift (lag) of both signals, expressed in number of weeks. Figures 9 and 10 contains displacements graphs of the stations considered in the paragraph.

\subsection{Deformation on GNSS stations located on an islands}

In this paragraph three stations located on an islands were taken into account. Two of them: MKEA and OUS2 were at a considerable distance from the nearest continents. Calculated displacements omit station's nearest loading mass which may affect the results of the modelled deformations. RESO station located on one of the islands of the Arctic Archipelago (Cornwallis Island) can behave (in terms of calculated deformations) like a station located inland or station located on the coast due to knowledge of the hydrological data in the nearest surrounding area. Assignment of RESO station to island group is somehow arbitrary, nevertheless we decided to show also the result for this site. In each case a small correlation coefficient was obtained along with a high values of signals shift. Modelled values of deformations for MKEA station are more than two times smaller than those observed. In case of OUS2 station modelled deformation does not exceed $1 \mathrm{~mm}$ despite clearly visible vertical movement, oscillating between the values of about $-8 \mathrm{~mm}$ to $10 \mathrm{~mm}$. Modelled displacements of the RESO station reaches very close values to those that are observed. Table 5 summarizes the main characteristics of the analysed signals. To describe the compatibility of the observed and modelled displacements selected correlation coefficient and mutual shift (lag) of both signals, expressed in number of weeks. Figure 11 contains displacements graphs of the stations considered in the paragraph. 

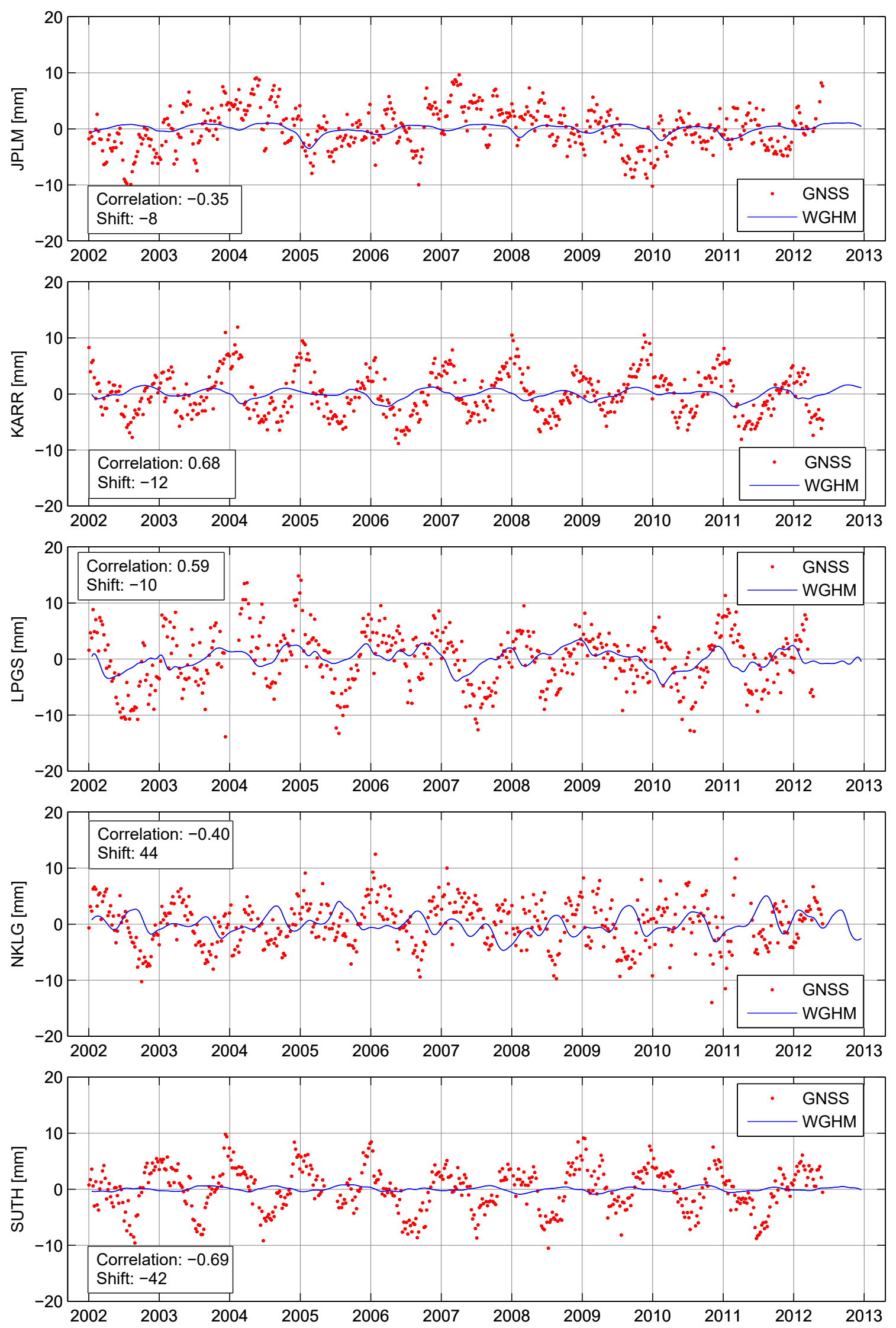

Fig. 9. Vertical displacements of the stations located near the shoreline: JPLM, KARR, LPGS, NKLG, SUTH. Graphs contains information about correlation of the observed and modelled signals and their mutual shift (lag) expressed in number of weeks 

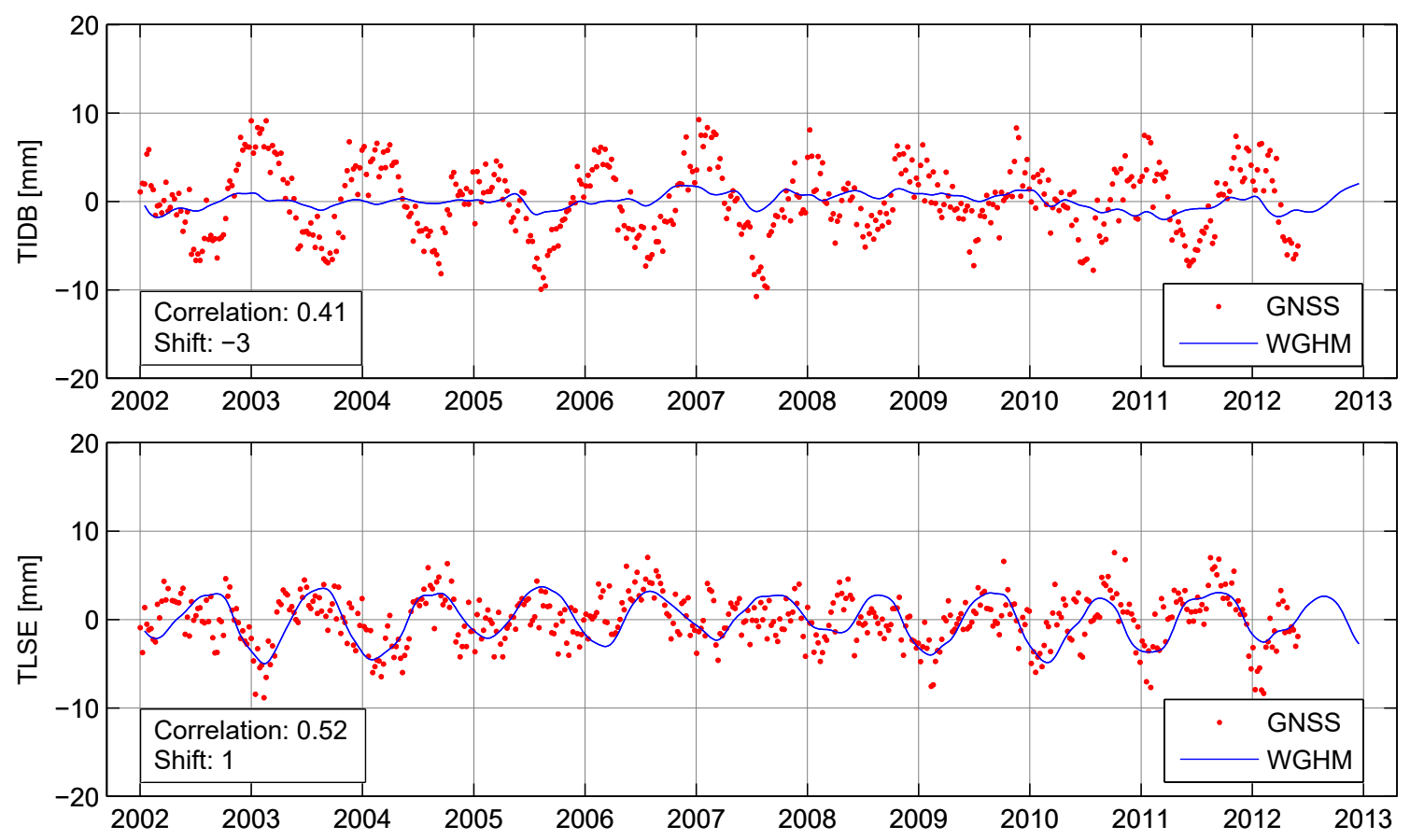

Fig. 10. Vertical displacements of the stations located near the shoreline: TIDB, TLSE. Graphs contains information about correlation of the observed and modelled signals and their mutual shift (lag) expressed in number of weeks

\section{Summary and conclusions}

Presented paper is dedicated to problems of deformation of the Earth's crust as a response to the loading caused by continental waters. Analysis on the compatibility of the observed and modelled vertical displacements were made on the basis of 23 monitoring stations assigned to one of three groups, determined by their localization and led to the following conclusions:

Applied calculation approach based on the dividing sphere's surface into zones with constant number of sectors, regardless of the latitude of calculated segment. The analyses showed that it was required to adopt shorter distances between adjacent zones than increasing number of segments in the zone (reducing the width of spherical figures).

Analysis of the accuracy of the results were made with programs: written by author, SPOTL and grat and showed a convergent results. Any differences depend on the way of dividing sectors into zones and adopted (source) values of the Green's function. However, they are not important enough to significantly improve the correlations of the observed and modelled displacements.

Value of correlation coefficient of the crustal displacements and the shift of two signals is highly dependent on the quality of the time series recorded at GNSS stations. Future work should take into account additional information such as e.g. related to earthquakes.

The study of the correlation of the observed and calculated displacements should cover a substantial part of the station included in the reference stations networks, such as IGS, which will allow to draw conclusions about the local and regional nature of the spatial deformation of the Earth's surface.

Crustal deformations in the vertical direction reach approximately tenfold higher values than deformation in the horizontal plane - among them higher values are achieved in the direction of meridian. 

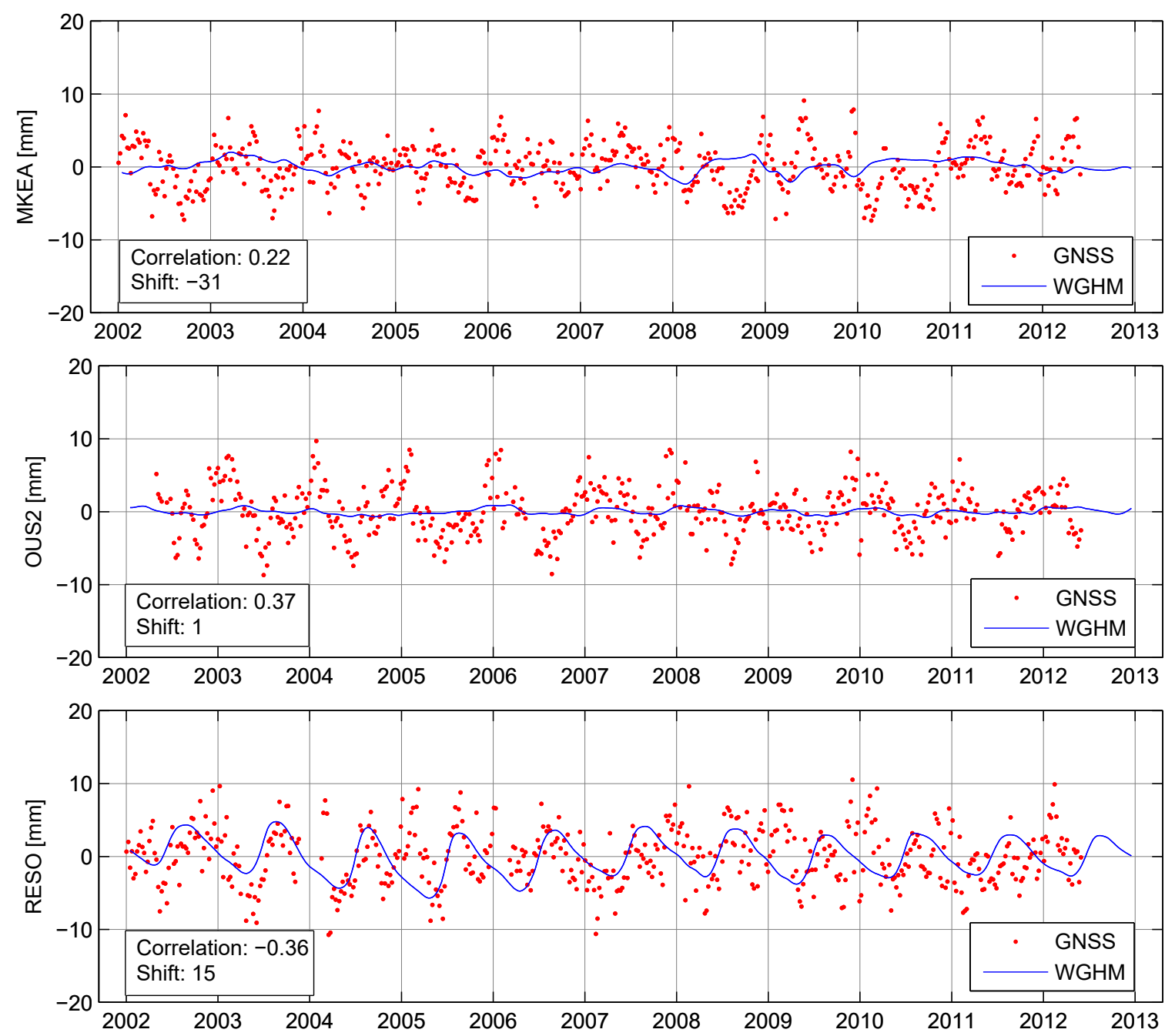

Fig. 11. Vertical displacements of the stations located on an island: MKEA, OUS2, RESO. Graphs contains information about correlation of the observed and modelled signals and their mutual shift (lag) expressed in number of weeks

In any case of the compared displacement for selected control stations, modelled values are smaller than observed, which leaves space for additional effects, which models are not included in the IERS Conventions.

For stations located inland, modelled and observed deformations are characterized by a good agreement. Introducing corrections to stations position will allow to remove a significant part of the station's movement.

Modelled displacement of RESO station, located on the one island of the Arctic Archipelago, are characterized by amplitudes and correlation coefficient typical for the stations located inland, which is probably due to maintaining hydrological data continuity on the area surrounding the station.

Time series of calculated displacements, for stations classified to groups other than inland, are characterized by much lower values in relation to the observed deformations, weaker correlation coefficients of signals and their higher shift. Further work, devoted to studying crustal deformation, should take into account additional deforming effects as the atmospheric load. 


\section{Acknowledgement}

Authors would like to acknowledge Dr. A. Güntner of GFZ, Potsdam, for providing WGHM data. Time series of GNSS stations vertical variations are based on IGS products. The source code of the program is made available to the public on the website http://www. grat.gik.pw . edu.pl/mzload/

\section{References}

Agnew, D. C. (2012). SPOTL: Some Programs for Ocean-Tide Loading. Technical Report. Retrieved from http://igppweb.ucsd.edu/ agnew/Spotl/spotlmain.html

Benavent, M., Arnoso, J., \& Montesinos, F. G. (2005). Computation of surface displacements, tilt and gravity variations due to ocean tide loading. Fisica de la Tierra, 97-112.

Döll, P., Alcamo, J., Henrichs, T., Kaspar, F., Lehner, B., Rösch, T., \& Siebert, S. (2001). The Global Integrated Water Model WaterGAP 2.1. EuroWasser: The global integrated water model WaterGAP 2.1, 2.1-2.18.

Döll, P., Kaspar, F., \& Lehner, B. (2003). A global hydrological model for deriving water availability indicators: model tuning and validation. Journal of Hydrology, 270(1-2), 105-134.

Dow, J. M., Neilan, R. E., \& Rizos, C. (2009, mar). The International GNSS Service in a changing landscape of Global Navigation Satellite Systems. Journal of Geodesy, 83, 191-198. doi: 10.1007/s00190-008-0300-3

Farrell, W. E. (1972). Deformation of the Earth by surface loads. Reviews of Geophysics and Space Physics, 10.3, 761-797.

Güntner, A., Stuck, J., Werth, S., Döll, P., Verzano, K., \& Merz, B. (2007). A global analysis of temporal and spatial variations in continental water storage. Water Resources Research, 43(W05416). doi: DOI:10.1029/2006WR005247

Hunger, M., \& Döll, P. (2008). Value of river discharge data for global-scale hydrological modeling. Hydrology and Earth System Sciences, 12, 841-861. Retrieved from http:// www.hydrol-earth-syst-sci.net/12/841/2008/hess-12-841-2008.html doi: 10.5194/hess-12-841-2008

Petit, G., \& Luzum, B. (2010). IERS Technical Note 36. 36 Frankfurt am Main: Verlag des Bundesamts für Kartographie und Geodäsie: IERS Convention Centre. Retrieved from http://www.iers.org/nn_11216/IERS/EN/Publications/TechnicalNotes/ tn36.html

Rajner, M. (2013, 9). Grat - open source software for computing atmospheric gravity correction numerical weather models. Potsdam. Retrieved from https://github .com/mrajner/grat

Rajner, M., \& Liwosz, T. (2011). Studies of crustal deformation due to hydrological loading on GPS height estimates. Geodesy and Cartography, 60.2, 137-146. doi: 10.2478/v10277-012-0012-y

Tervo, M., Virtanen, H., \& Bilker-Koivula, M. (2006). Environmental loading effects on GPS time series. Bull. d'Inf. Marées Terr, 142(11), 407-11.

van Dam, T., Plag, H.-P., Francis, O., \& Gegout, P. (2003). Proceedings of the IERS Workshop on Combination Research and Global Geophysical Fluids (Vol. IERS Technical Note 30). Munich, Germany: Bavarian Academy of Sciences. Retrieved from http:// www. iers.org/nn_11216/IERS/EN/Publications/TechnicalNotes/tn30.html

van Dam, T., Wahr, J., Milly, P., Shmakin, A., Blewitt, G., Lavallée, D., \& Larson, K. (2001, feb). Crustal displacements due to continental water loading. Geophysical Research Letter, No 28, 651-654. doi: 10.1029/2000GL012120 
Authors: $\quad$ Michał Zygmunt m.zygmunt87@wp.pl

Marcin Rajner mrajner@gik.pw .edu.pl

Tomasz Liwosz tliwosz@gik.pw.edu.pl

Warsaw University of Technology

Department of Geodesy and Geodetic Astronomy

pl. Politechniki 1, Warszawa, Poland 